\title{
Séquestration du Carbone et Politique Climatique Optimale
}

\author{
André Grimaud ${ }^{1}$, and Luc Rouge ${ }^{23}$
}

17 octobre 2008

\footnotetext{
${ }^{1}$ Toulouse School of Economics (IDEI and LERNA), Manufacture des Tabacs, 21 Allée de Brienne, 31000 Toulouse, France, and Toulouse Business School. E-mail : grimaud@cict.fr

${ }^{2}$ Corresponding author. Toulouse Business School, 20 Bd Lascrosses, 31068 Toulouse Cedex 7, France. E-mail : l.rouge@esc-toulouse.fr Tel : +33 561294820 Fax : +335612949 94

${ }^{3}$ Les auteurs tiennent à remercier Bertrand Magné pour ses commentaires et ses suggestions.
} 


\section{Résumé}

La séquestration du carbone émis lors de la combustion de ressources fossiles est une option qui suscite aujourd'hui beaucoup d'intérêt; elle peut en effet contribuer à la décarbonisation progressive de l'économie dans le cadre de la lutte contre le changement climatique. Ce procédé, dont la viabilité est à présent établie (IPCC, 2005), contient deux phases : la captation du carbone, qui sépare le $\mathrm{CO}_{2}$ des autres émissions, puis le stockage dans des réservoirs (que ce soient des gisements d'hydrocarbures épuisés ou des aquifères marins profonds, par exemple).

S'il existe aujourd'hui une importante littérature sur la question de l'utilisation à long terme de ressources non-renouvelables polluantes, force est de constater qu'elle néglige généralement l'existence d'une telle technologie. L'objectif de cet article est d'étudier en quoi sa prise en compte modifie et complète les principaux résultats de cette littérature. Nous présentons pour cela un modèle de croissance endogène dans lequel l'utilisation d'une ressource naturelle nonrenouvelable et polluante génère des émissions de $\mathrm{CO}_{2}$, dont le stock accumulé affecte négativement à la fois l'utilité des ménages et la productivité des entreprises. Nous montrons que, si la séquestration accélère le rythme optimal d'extraction, elle peut aussi générer de plus fortes émissions de $\mathrm{CO}_{2}$ pour les premières générations. De plus, elle est défavorable à la croissance de l'output.

Notons ici que l'existence d'une telle technologie rompt le lien systématique, généralement fait dans la littérature, entre utilisation de la ressource et émissions polluantes. En effet, puisque la technologie de séquestration permet de réduire la pollution effective pour chaque unité de ressource utilisée, l'extraction et la pollution sont partiellement déconnectées. Dès lors, la politique climatique que nous mettons en œuvre consiste en une taxe sur les émissions de carbone, et non sur la ressource. Contrairement à de précédents résultats de la littérature, le niveau de cette taxe compte ici, puisqu'il donne les bonnes incitations à l'effort de séquestration. Par ailleurs, le taux de croissance optimal de cette taxe carbone est positif; nous montrons néanmoins que cet instrument de politique climatique peut être interprété ex-post comme une taxe ad-valorem décroissante sur la ressource, ce qui fait le lien avec la littérature existante.

Mots-clefs : croissance endogène, politique climatique, pollution, ressources non-renouvelables, séquestration du carbone.

Classification JEL : O32, O41, Q20, Q32 


\section{Introduction}

La possibilité de capter et de séquestrer une partie du dioxyde de carbone émis lors de la combustion des énergies fossiles a récemment suscité beaucoup d'intérêt, d'autant que la viabilité d'une telle technique est maintenant établie (pour un survol de cette question, voir le rapport spécial de l'IPCC, 2005). Hoffert et al., 2002, notamment, soulignent que la séquestration du carbone, c'est-à-dire la réduction de la quantité de carbone émise par unité de ressource fossile utilisée, est un élément-clef dans le processus global de décarbonisation.

Cette séquestration s'opère tout d'abord à travers la captation du carbone, activité à laquelle on fait parfois référence sous l'appellation contrôle des émissions (voir Kolstad et Toman, 2001) : ce processus consiste à séparer le dioxyde de carbone des autres émissions de gaz lors de la production d'énergie. Il est particulièrement adapté aux centrales électriques de grande taille mais peut aussi être utilisé dans le cadre de l'énergie non électrique ${ }^{1}$. Une fois capturés, les gaz sont alors stockés dans différents réservoirs. Ces réservoirs potentiels comprennent notamment des gisements d'hydrocarbures (gaz ou pétrole) épuisés, des veines de charbon non exploitables, ou encore des aquifères salins profonds. Ces différents lieux de stockage se distinguent à la fois par leurs capacités, leurs coûts d'accès, et leur aptitude à conserver durablement le carbone.

Malgré les multiples incertitudes pesant sur la possibilité d'un déploiement significatif des activités de séquestration, notamment en ce qui concerne l'impact environnemental d'injections massives de carbone, cette technologie apparaît aujourd'hui comme une option prometteuse pour les industries d'extraction de ressources fossiles. Le coût estimé de la captation du carbone va de 40 à 90 dollars par tonne de $\mathrm{CO}_{2}$ captée et stockée (AIE, 2006). Selon les technologies utilisées, ceci impliquerait un accroissement de 25 à $45 \%$ du coût de l'électricité. Les prévisions de l'AIE indiquent que l'utilisation de technologies de séquestration du carbone représentera 20 à $28 \%$ de la réduction des émissions de $\mathrm{CO}_{2}$ en $2050: 6500$ à 7500 millions de tonnes de $\mathrm{CO}_{2}$ émises pourraient ainsi être évitées, dont $60 \%$ dans le seul secteur électrique. Simultanément, l'utilisation du charbon augmenterait alors de $13 \%$ à $32 \%$ par rapport à son niveau actuel.

Si la question de l'utilisation à long-terme de ressources non-renouvelable polluantes comme les ressources fossiles a donné lieu à de nombreuses études, celles-ci ont jusqu'ici généralement négligé la possibilité de séquestrer le carbone. Au vu de l'importance d'une telle option, il serait pourtant intéressant de la prendre en compte, et d'analyser en quoi les principaux résultats de ces travaux sont modifiés lorsque la séquestration du carbone est un choix techniquement

\footnotetext{
${ }^{1}$ L'hydrogène produit lors de ce processus pourrait ensuite alimenter les besoins énergétiques du secteur des transports à l'aide de piles à combustible.
} 
viable. Rappelons ici brièvement les principaux résultats de la littérature standard (c'est-à-dire sans séquestration). Les deux questions principales sont : l'optimum social d'une part, et sa mise en oeuvre dans une économie décentralisée ainsi que l'impact des politiques environnementales d'autre part. Que ces questions soient étudiées dans un cadre d'équilibre partiel (par exemple, Sinclair, 1992, 1994, Withagen, 1994, Ulph et Ulph, 1994, ou Tahvonen, 1997) ou dans des modèles de croissance en équilibre général (Schou, 2000, 2002, Grimaud et Rouge, 2005, 2008, ou Groth et Schou, 2007), les auteurs montrent généralement qu'à l'optimum l'extraction de la ressource, et donc les émissions polluantes, doivent être retardées. Les recommandations de ces modèles en termes de politique environnementale sont, elles, moins unanimes. Par exemple, Sinclair, 1994, plaide pour une taxe ad valorem décroissante sur l'utilisation de la ressource, tandis que Ulph et Ulph, 1994, parmi d'autres, montrent qu'une telle taxe ne sera pas toujours optimale, et particulièrement quand le stock de pollution se dégrade partiellement (ou, de façon équivalente, quand l'environnement s'auto-régénère partiellement). Si l'on considère uniquement les modèles de croissance endogène avec des ressources non-renouvelables polluantes, à l'exception de Schou, 2000, 2002, pour qui une politique environnementale est inutile, les résultats montrent généralement que la taxe ad valorem optimale sur la ressource est décroissante (voir Grimaud et Rouge, 2005, 2008, ou Groth et Schou, 2007). De plus, comme dans Sinclair, 1994, une modification du niveau de la taxe a uniquement des effets redistributifs : elle n'a pas d'impact sur la dynamique du modèle, en particulier sur les sentiers d'extraction de la ressource et d'émissions polluantes. Il est important de souligner ici que toutes ces études font un lien systématique, sous la forme d'une relation fonctionnelle simple, généralement linéaire, entre l'extraction de la ressource et les émissions de gaz à effet de serre. Il est donc équivalent de taxer les émissions polluantes ou la ressource elle-même. Dès lors que la possibilité de séquestrer une partie du carbone émis lors de la combustion des ressources fossiles est prise en compte, ce lien est partiellement rompu. En effet, la possibilité de séquestrer une partie des émissions de $\mathrm{CO}_{2}$ causées par la combustion de ressources fossiles déconnecte l'utilisation de la ressource de la pollution effective. Nous souhaitons ici étudier l'existence d'une telle technologie dans le cadre d'un modèle de croissance endogène avec ressources non-renouvelables polluantes, et analyser en quoi les principaux résultats de la littérature mentionnés plus haut, notamment ceux concernant la politique climatique optimale sont modifiés dans un tel contexte ${ }^{2}$.

\footnotetext{
${ }^{2}$ De nombreux travaux ont étudié les effets d'une réduction de la pollution (pollution abatement) dans des modèles avec préférences environnementales et croissance (par exemple, Smulders et Gradus, 1996). En relation avec cette littérature, on peut considérer la technologie de séquestration comme une possibilité importante de réduction de la pollution. Cette question a été abordée dans plusieurs études empiriques sur le changement climatique (par exemple, Gerlagh et van der Zwaan, 2006) mais, à notre connaissance, elle n'a jamais été étudiée dans le cadre de modèles théoriques de croissance endogène.
} 
Nous présentons un modèle de croissance endogène dans lequel la production d'un bien de consommation requiert l'utilisation d'une ressource naturelle extraite d'un stock fini. De plus, ceci génère des émissions polluantes, que l'on peut interpréter comme des émissions de gaz à effet de serre (et plus particulièrement de carbone), dont le flux vient grossir le stock déjà présent dans l'atmosphère. Ce stock de carbone connaît par ailleurs une dégénérescence naturelle partielle. Enfin, il est à la fois un argument de la fonction d'utilité des ménages (comme dans Schou, 2002, ou Grimaud et Rouge, 2008, par exemple) et de la fonction de production du bien de consommation (comme par exemple dans Popp, 2004, ou Schou, 2000). Mais la principale nouveauté dans ce modèle réside dans la prise en compte de l'existence d'une technologie qui, à partir d'un effort donné, permet de séquestrer une partie du carbone émis. Dès lors, nous distinguons bien le $\mathrm{CO}_{2}$ total potentiellement émis lors de la combustion d'une unité de ressource (que nous appellerons dorénavant le carbone potentiel total par unité de ressource) de l'émission réelle, c'est-à-dire, la pollution restante après séquestration. L'implication en termes de politique climatique est alors immédiate : l'optimum de premier rang ne peut être restauré qu'en taxant la pollution, et non la ressource elle-même ${ }^{3}$. Notons enfin que, dans un premier temps, nous présentons le modèle dans une version non spécifiée, ce qui nous permet d'obtenir des résultats généraux, notamment en ce qui concerne la formulation de la politique climatique optimale. Dans un second temps, nous proposons une version spécifiée, qui permet d'étudier plus précisément la dynamique de l'économie.

Par ailleurs, puisque nous concentrons notre étude sur l'impact de la séquestration du carbone sur les trajectoires de l'économie décentralisée et sur les trajectoires optimales, ainsi que sur la définition d'une politique climatique optimale, nous ferons les hypothèses suivantes. Tout d'abord, sans perte de généralité, nous ne distinguerons pas la phase de captation du $\mathrm{CO}_{2}$ de celle de l'injection. Nous ferons également l'hypothèse que les puits de carbone (aquifères marins profonds, océans...) ont une contenance suffisamment élevée pour accueillir toute quantité de carbone séquestré, et donc que le développement de la technologie n'est pas contraint par la capacité de stockage. Enfin, pour la simplicité de l'exposé, nous supposerons que la séquestration du carbone s'applique à l'utilisation de toute ressource fossile, quel que soit son usage, du moment qu'il est destiné à la production d'énergie.

Les principaux résultats de cette étude peuvent être résumés comme suit. L'existence d'une

\footnotetext{
${ }^{3}$ Ici nous supposons que le régulateur est capable de mesurer les émissions de carbone. Ce n'est pas forcément toujours le cas : bien que les données sur les émissions soient assez fiables dans les pays industrialisés, la collecte de données précises sur les activités industrielles et la déduction des émissions qui en résultent peut s'avérer difficile dans des régions en développement.
} 
technologie de séquestration du carbone accélère le rythme optimal d'extraction de la ressource, tout en relâchant la contrainte environnementale pour les ménages. De plus, elle modifie le sentier d'émissions de $\mathrm{CO}_{2}$ de la façon suivante. A long-terme, le niveau de la pollution décroît sans ambigüité. Cependant, si les préférences environnementales ne sont pas suffisamment fortes, le niveau de pollution peut être accru à court-terme. Dans ce cas, nous avons le résultat contre-intuitif suivant : l'existence d'une technologie de séquestration du carbone conduit à une augmentation des émissions de $\mathrm{CO}_{2}$ pour les premières générations. Enfin, une telle technologie s'avère négative pour la croissance de l'output, ceci étant dû à l'effet combiné de l'accélération de l'extraction et d'un effet négatif sur l'effort de R\&D.

Dans ce cadre d'analyse, comme nous l'avons déjà mentionné, une taxe sur la pollution n'est plus équivalente à une taxe sur la ressource; ce sont bien les émissions qui doivent être taxées si l'on souhaite des résultats de premier rang. Par ailleurs, contrairement aux résultats obtenus dans des contextes sans séquestration, comme dans Sinclair, 1994, ou Grimaud et Rouge, 2005, 2008, par exemple, ici, le niveau de la taxe importe, en particulier parce qu'il conduit à mettre en oeuvre l'effort de séquestration optimal. Nous montrons également qu'un accroissement de la taxe conduit l'économie à retarder l'extraction (ce qui, ici, nous ramène à la littérature standard présentée plus haut), et modifie également la quantité de pollution émise par unité de ressource utilisée.

Enfin, nous calculons la trajectoire optimale de la taxe, dont le taux de croissance est positif (ceci provient de la décroissance de l'utilité marginale de la consommation). Nous montrons également que cette taxe peut être exprimée ex-post comme une taxe ad-valorem décroissante sur la ressource.

La suite de cet article est organisée de la façon suivante. Le modèle, dans sa forme non spécifiée, est présenté dans la première partie. Dans la deuxième partie, nous caractérisons l'équilibre de l'économie décentralisée, puis nous utilisons la version spécifiée du modèle pour analyser l'impact de la politique climatique. La troisième partie est consacrée à l'analyse de bienêtre et à l'étude de la politique climatique optimale. Nous faisons quelques remarques conclusives dans la dernière partie.

\section{Le Modèle}

Nous présentons tout d'abord une version non spécifiée du modèle, afin d'obtenir des conditions générales d'optimalité et d'équilibre, de les comparer, et de définir une formulation générale 
de la politique climatique optimale (voir également les sections suivantes).

A tout instant $t$, les entreprises produisent une quantité $Y_{t}$ d'un bien de consommation suivant la technologie :

$$
Y_{t}=F\left(A_{t}, L_{Y_{t}}, R_{t}, X_{t}\right)
$$

où $L_{Y t}$ est la quantité de travail consacrée à la production, $A_{t}$ est un stock de connaissance, $R_{t}$ est le flux de ressource non-renouvelable utilisé dans le processus de production, et $X_{t}$ est le stock de carbone accumulé dans l'atmosphère. Nous noterons $F_{L}, F_{A}, F_{R}$ et $F_{X}(<0)$ les productivités marginales.

Nous considérons que chaque innovation est un bien public, indivisible et à durée de vie infinie, qui est simultanément utilisé par le secteur produisant le bien de consommation et le secteur de la recherche ${ }^{4}$. Formellement, il s'agit d'un point sur le segment $\left[0, A_{t}\right]$. A toute date $t$, la technologie de production de la connaissance est :

$$
\dot{A}_{t}=h\left(A_{t}, L_{R D t}\right)
$$

où $L_{R D t}$ est la quantité de travail consacrée à la $\mathrm{R} \& \mathrm{D}$. Nous noterons $h_{A}$ et $h_{L}$ les productivités marginales.

La pollution est générée par l'utilisation de la resource non-renouvelable dans le processus de production. En l'absence d'une activité de séquestration du carbone, le flux de pollution serait une fonction linéaire du flux de ressource utilisée : $\gamma R_{t}$, où $\gamma>0$. Ainsi, $\gamma R_{t}$ peut être vu comme le contenu total en carbone de la ressource extraite ou, de façon équivalente, comme le potentiel polluant maximum de l'entreprise produisant le bien de consommation. Néanmoins, l'entreprise peut stocker une partie de ce carbone. Dès lors, le flux de pollution réellement émise est :

$$
P_{t}=\gamma R_{t}-Q_{t}
$$

où $Q_{t}$ est la quantité de carbone séquestrée. Nous supposons qu'elle est produite à partir de deux inputs : le contenu en carbone $\gamma R_{t}$ via la quantité de ressource extraite $R_{t}$, et le flux de travail $l_{t}$. La technologie de séquestration est :

$$
Q_{t}=q\left(\gamma R_{t}, l_{t}\right)
$$

Nous noterons $q_{\gamma R}$ et $q_{l}$ les productivités marginales.

\footnotetext{
${ }^{4}$ Par exemple, nous pouvons penser à un rapport scientifique présentant une théorie nouvelle qui pourra être utilisée à la fois dans la production et dans l'activité de recherche.
} 
Commentons brièvement la forme de cette fonction de séquestration. Pour toute quantité de carbone potentiellement émis $\gamma R_{t}$, c'est uniquement l'effort en termes de travail qui permet le stockage du carbone. Bien entendu, nous pourrions également prendre en compte le capital physique, par exemple. Néanmoins, en ajoutant une nouvelle variable d'état, cela complexifierait considérablement l'analyse.

Le carbone s'accumule dans l'atmosphère; le flux de carbone émis $\left(P_{t}\right)$ vient donc grossir le stock pré-existant $X_{t}$. On suppose que $X_{t}=X_{0}+\int_{0}^{t} P_{s} e^{\theta(s-t)} d s$, ce qui donne la loi d'évolution suivante :

$$
\dot{X}_{t}=\theta\left(X_{0}-X_{t}\right)+P_{t}
$$

où $\theta$ est le taux positif (supposé constant) de dégénérescence de ce stock, ou encore de régénération de l'atmosphère.

La ressource non-renouvelable est extraite à coût nul d'un stock initial $S_{0}$ fini. Nous avons la loi de mouvement standard du stock de ressource :

$$
\dot{S}_{t}=-R_{t}
$$

La production du bien de consommation est entièrement consommée par le ménage représentatif :

$$
Y_{t}=C_{t}
$$

Nous supposons que la population est constante, normalisée à 1, et que chaque individu est doté d'une unité de travail. Nous avons donc :

$$
1=L_{Y t}+L_{R D t}+l_{t}
$$

L'utilité instantanée du ménage représentatif dépend de la consommation $C_{t}$ mais également du stock de carbone présent dans l'atmosphère, $X_{t}$. Nous écrivons la fonction d'utilité intertemporelle ainsi :

$$
\mathbf{U}=\int_{0}^{+\infty} U\left(C_{t}, X_{t}\right) e^{-\rho t} d t, \rho>0
$$

où les dérivées partielles de la fonction d'utilité par rapport à la consommation et à la pollution sont notées $U_{C}(>0)$ et $U_{X}(<0)$, et sont respectivement positive et négative. Notons que, dans ce modèle, le stock de carbone accumulé affecte à la fois les ménages (équation 9) et la production (équation 1). 


\section{Equilibre de l'économie décentralisée et impact de la politique climatique}

Nous commençons ici par l'étude de la version non spécifiée du modèle présentée dans la section précédente. Le prix du bien $Y$ est normalisé à 1 , et $w_{t}, p_{t}^{R}$ et $r_{t}$ sont, respectivement, le salaire, le prix de la ressource, et le taux d'intérêt sur un marché financier parfait. Afin d'éliminer les défaillances de marché provenant du fait que les entreprises ne prennent pas en compte l'externalité négative consécutive à l'utilisation de la ressource non-renouvelable lors de la production du bien de consommation, c'est-à-dire les émissions de $\mathrm{CO}_{2}$, nous mettons en place une politique économique, que nous appellerons la politique climatique. Il s'agit d'une taxe sur ces émissions. Notons que la politique climatique consiste bien en une taxe sur la pollution, et non sur la ressource polluante, comme dans Grimaud et Rouge, 2005, 2008, ou Groth et Schou, 2007. En effet, ce sont les émissions de carbone qui constituent l'externalité fondamentale. Puisqu'une technologie de séquestration du carbone est disponible, une taxe sur ces émissions et une taxe sur la ressource polluante ne sont plus équivalentes.

Nous allons voir ci-dessous que la taxe sur les émissions de carbone a deux effets principaux : d'une part, elle conduit à retarder l'extraction de la ressource (comme dans les modèles sans séquestration); d'autre part, elle crée des incitations à produire des efforts dans la séquestration.

\subsection{Comportements des agents}

Notre objectif ici n'étant pas d'analyser l'impact d'autres distorsions que les émissions de $\mathrm{CO}_{2}$, nous supposons que la recherche est financée de façon optimale (au moyen d'une éventuelle intervention publique, comme nous le précisons plus bas). Il reste donc une seule distorsion, la pollution, et un seul outil de politique économique, la taxe carbone.

\section{Secteur du bien de consommation :}

A toute date t, le profit de l'entreprise est

$$
\pi_{t}^{Y}=F\left(L_{Y t}, A_{t}, R_{t}, X_{t}\right)-w_{t}\left(L_{Y t}+l_{t}\right)-p_{R t} R_{t}-\tau_{t}\left[\gamma R_{t}-q\left(\gamma R_{t}, l_{t}\right)\right]
$$

où $\tau_{t}$ est la taxe unitaire sur les émissions de carbone. On obtient les trois conditions de premier ordre suivantes :

$$
\begin{gathered}
F_{L}=w_{t}, \\
F_{R}=p_{R t}+\tau_{t} \gamma\left(1-q_{\gamma_{R}}\right),
\end{gathered}
$$




$$
\tau_{t} q_{l}=w_{t}
$$

\section{Secteur de la R\&D :}

Afin de formaliser le marché de la connaissance, nous procédons de la façon suivante. Nous calculons la valeur sociale de chaque innovation, qui est la somme des dispositions à payer de tous les utilisateurs (c'est-à-dire la somme des prix de Lindahl). Puis, nous supposons que l'entreprise innovante reçoit cette valeur sociale.

L'absence de biens intermédiaires est ici une pure convention qui permet d'alléger les calculs. Nous savons que dans le modèle de différenciation horizontale de Romer, la recherche est sousfinancée. Par contre, dans le modèle de différenciation verticale d'Aghion et Howitt, elle peut être sous ou sur-financée. Par ailleurs, Benassy, 1998, a montré qu'une modification minime du modèle de Romer conduit au même résultat qu'Aghion et Howitt, 1998. Enfin, plusieurs études empiriques (Jones et Williams, 1998, par exemple) montrent que la recherche est généralement sous-financée : la valeur privée des innovations (c'est-à-dire celle qui est payée à l'innovateur) représente environ un quart à un tiers de leur valeur sociale (définie ci-dessus). Notre modélisation peut être interprétée comme la forme réduite d'un modèle plus standard de croissance endogène avec biens intermédiaires dans lequel les autorités subventionnent la recherche de façon optimale, afin que l'innovateur reçoive la totalité de la valeur sociale de son innovation (voir sur ce point Barro et Sala-i-Martin, 2005).

A toute date $t$, la valeur d'une innovation est :

$$
V_{t}=\int_{t}^{+\infty} v_{s} e^{-\int_{t}^{s} r_{u} d u} d s
$$

où $v_{s}$ est la somme des dispositions à payer du secteur du bien de consommation $\left(v_{s}^{Y}\right)$ et du secteur de la $\operatorname{R\& D}\left(v_{s}^{R D}\right): v_{s}=v_{s}^{Y}+v_{s}^{R D}$.

Le profit sur les innovations produites à la date $t$ est :

$$
\pi_{t}^{R D}=h\left(A_{t}, L_{R D t}\right) V_{t}-w_{t} L_{R D t} .
$$

La maximisation de ce profit par rapport à $L_{R D t}$ conduit à la condition de premier ordre suivante :

$$
h_{L} V_{t}=w_{t}
$$


Des formules (10) et (15), nous avons :

$$
\begin{gathered}
v_{t}^{Y}=\frac{\partial \pi_{t}^{Y}}{\partial A_{t}}=F_{A} \\
\text { et } v_{t}^{R D}=\frac{\partial \pi_{t}^{R D}}{\partial A_{t}}=h_{A} V_{t},
\end{gathered}
$$

ce qui donne la valeur sociale d'une innovation :

$$
v_{t}=F_{A}+h_{A} V_{t}
$$

\section{Ménage représentatif :}

A toute date $t$, le ménage représentatif maximise la fonction d'utilité $\int_{0}^{+\infty} U\left(C_{t}, X_{t}\right) e^{-\rho t} d t$ sous la contrainte $\dot{B}_{t}=w_{t}+r_{t} B_{t}+p_{R t} R_{t}-T_{t}-C_{t}$, où $B_{t}$ est le stock de titres en $t$, et $T_{t}$ est une taxe (subvention) forfaitaire du gouvernement. Cette maximisation conduit à la condition habituelle suivante :

$$
\rho-\frac{\dot{U}_{C}}{U_{C}}=r_{t}
$$

\section{Secteur de la ressource :}

Sur le marché de la ressource, supposé concurrentiel, la maximisation de la fonction de profit $\int_{t}^{+\infty} p_{R s} R_{s} e^{-\int_{t}^{s} r_{u} d u} d s$, sous la contrainte $\dot{S}_{s}=-R_{s}, S_{s} \geq 0, R_{s} \geq 0, s \geq t$, conduit à la "règle d'Hotelling" d'équilibre standard :

$$
\frac{\dot{p}_{R t}}{p_{R t}}=r_{t}, \text { pour tout t. }
$$

\subsection{Equilibre}

Notre objectif est maintenant de présenter les trois conditions caractéristiques de l'équilibre (vérifiées à chaque date $t$ ). Ces conditions pourront être rapprochées des conditions caractéristiques de l'optimum social, présentées dans la section suivante.

Proposition 1 Les conditions caractéristiques de l'économie à l'équilibre décentralisé sont (nous omettons les indices temporels par commodité de notation) :

$$
\begin{gathered}
\rho-\frac{\dot{U}_{C}}{U_{C}}=\frac{F_{A} h_{L}}{F_{L}}+h_{A}+\frac{\dot{F}_{L}}{F_{L}}-\frac{\dot{h}_{L}}{h_{L}}, \\
\frac{F_{L}}{q_{l}}=\tau
\end{gathered}
$$


et

$$
F_{R}=p_{R}+\tau \gamma\left(1-q_{\gamma R}\right)
$$

Preuve. En différenciant (14) et (16) par rapport au temps, on obtient $\frac{\dot{w}_{t}}{w_{t}}=r_{t}-\frac{v_{t}}{V_{t}}+\frac{\dot{q}_{L}}{q_{L}}$. En utilisant (11), (16) et (17), on obtient $: r_{t}=\frac{\dot{F}_{L}}{F_{L}}-\frac{\dot{q}_{L}}{q_{L}}+\frac{F_{A} q_{L}}{F_{L}}+q_{A}$. En combinant ce résultat avec (18), on obtient (20).

En combinant les équations (12) et (13), on obtient (21).

Enfin, (12) donne directement (22).

L'équation (20) est une version modifiée de la condition habituelle de Keynes-Ramsey. En effet, contrairement au modèle de croissance standard, il n'y a pas ici de stock de capital physique mais un stock $A_{t}$ de connaissance : ceci explique que la productivité marginale du capital est remplacée par le membre de droite dans (20). La complexité relative de cette expression vient du fait qu'une variation de $C_{t}$ est compensée par un transfert de travail entre les secteurs du bien de consommation $\left(L_{Y}\right)$ et de la recherche $\left(L_{R D}\right)$, ce qui modifie toute la trajectoire de la variable $A_{t}$.

L'équation (21) montre l'impact de la taxe carbone sur le choix de séquestration des entreprises : la taxe est égale au coût, exprimé en termes de bien, d'une unité de carbone séquestrée.

Enfin, l'équation (22) est une version modifiée de la condition habituelle d'Hotelling, dans laquelle le membre de droite représente le coût généralisé (taxe carbone comprise) d'une unité de ressource.

\subsection{Spécification}

Afin d'aller plus loin dans cette analyse, nous retenons à présent les formes fonctionnelles suivantes :

$$
\begin{gathered}
Y_{t}=A_{t}^{\nu} L_{Y t}^{\alpha} R_{t}^{1-\alpha} e^{-\beta X_{t}}, \beta>0, \\
\dot{A}_{t}=\delta A_{t} L_{R D t}, \delta>0, \\
Q_{t}=\left(\gamma R_{t}\right)^{\eta} l_{t}^{1-\eta}, 0<\eta<1, \text { si } l_{t}<\gamma R_{t},
\end{gathered}
$$

et

$$
Q_{t}=\gamma R_{t} \text {, si } l_{t} \geq \gamma R_{t}
$$

Le flux de pollution est entièrement séquestré dès lors que $l_{t} \geq \gamma R_{t}$. Pour tout $\gamma R_{t}$, le coût en termes de travail, $l_{t}=Q_{t}^{1 /(1-\eta)}\left(\gamma R_{t}\right)^{-\eta /(1-\eta)}$, est une fonction croissante et convexe de $Q_{t}$. Le coût moyen et le coût marginal, respectivement $l_{t} / Q_{t}=Q_{t}^{\eta /(1-\eta)}\left(\gamma R_{t}\right)^{-\eta /(1-\eta)}$ et $\partial l_{t} / \partial Q_{t}=$ 
$[1 /(1-\eta)] Q_{t}^{\eta /(1-\eta)}\left(\gamma R_{t}\right)^{-\eta /(1-\eta)}$, sont également des fonctions croissantes de $Q_{t}$. Nous choisissons une forme Cobb-Douglas pour cette technologie de séquestration car elle permet des développements analytiques simples.

Enfin, l'utilité instantanée prend la forme :

$$
U\left(C_{t}, X_{t}\right)=\ln C_{t}-\omega X_{t}, \omega>0 .
$$

L'utilité marginale instantanée du stock de carbone, $-\omega$, est ici constante, contrairement à l'hypothèse faite par Aghion et Howitt, 1998, par exemple. Dans le cas de dommages environnementaux sévères, il serait peut-être plus réaliste de considérer que la désutilité marginale est croissante (comme, par exemple, dans le cas de catastrophes naturelles). Néanmoins, cette hypothèse permet des calculs simplifiés lorsqu'on analyse la dynamique de transition dans le cadre d'un modèle d'équilibre général.

Il n'est pas possible, pour des raisons évidentes, d'étudier l'impact sur l'équilibre de tous les profils possibles de taxes carbone. Nous allons restreindre notre analyse à certains profils particuliers. Nous montrons plus bas que la taxe optimale (exprimée en termes de bien $Y_{t}$ ) a pour expression : $-\left(1 / U_{C}\right) \int_{t}^{\infty}\left(U_{C} F_{X}+U_{X}\right) e^{-(\rho+\theta)(s-t)} d s$. Puisque $U_{C}=1 / C_{t}$, la taxe optimale dépend donc linéairement de $C_{t}=Y_{t}$. Dès lors, nous allons, dans la section présente, étudier l'impact d'une politique environnementale consistant en une taxe croissant au même rythme que l'output : $\tau_{t}=a Y_{t}$ (où $a$ est une constante).

Nous noterons $g_{Z_{t}}$ le taux de croissance de toute variable $Z_{t}$ à la date $t$. Par ailleurs, l'indice supérieur ${ }^{e}$ caractérise une variable à l'équilibre décentralisé.

Proposition 2 A l'équilibre de l'économie décentralisée, en présence d'une taxe carbone strictement positive (c'est-à-dire $\tau>0$ ) croissant au même rythme que l'output, à toute date $t$ :

(i) L'économie est toujours en transition.

(ii) Les flux d'extraction de ressource $\left(R_{t}^{e}\right)$, de séquestration $\left(Q_{t}^{e}\right)$ et de pollution $\left(P_{t}^{e}\right)$ décroissent avec le temps.

(iii) Le travail dans la production de bien de consommation $\left(L_{Y}^{e}\right)$ est constant dans le temps. Le travail dans la séquestration $\left(l_{t}^{e}\right)$ est proportionnel au flux de ressource extraite $\left(R_{t}^{e}\right)$, et suit donc la même dynamique $: g_{l_{t}}^{e}=g_{R t}^{e}<0$. Dès lors, le travail dans la recherche $\left(L_{R D t}^{e}\right)$ crồt au cours du temps et converge à l'infini vers le niveau constant $1-L_{Y}^{e}$.

Les niveaux et les taux de croissance des différentes variables sont donnés dans l'Annexe 1.

Preuve. Voir Annexe 1. 
Supposons qu'aucune politique climatique n'est mise en place (soit $\tau=0$ à tout instant). Ici, l'économie saute directement à son état-stationnaire, dans lequel la quantité de travail consacrée à la séquestration est nulle : $l^{e}=0$, ce qui signifie qu'il n'y a aucun stockage de carbone $\left(Q^{e}=0\right)$. Ceci implique également que le $\mathrm{CO}_{2}$ potentiel est intégralement émis dans l'atmosphère, c'est-àdire $P^{e}=\gamma R^{e}$. Par ailleurs, le travail utilisé dans la production du bien de consommation, $L_{Y}^{e}$, est constant, et le travail consacré à la recherche, $L_{R D}^{e}=1-L_{Y}^{e}$ l'est donc également. Le flux d'extraction à la date $t$ est $R_{t}^{e}=\rho S_{0} e^{-\rho t}$. Ceci implique $g_{R}^{e}=-\rho$ pour tout $t$. Ce dernier cas correspond à l'optimum sans pollution (et donc sans séquestration), comme nous le montrons plus bas. Nous revenons également plus bas sur les résultats présentés dans cette proposition.

\subsection{Impact de la politique climatique}

Nous étudions à présent les effets de la politique climatique sur la trajectoire de l'économie à l'équilibre décentralisé.

Proposition 3 Un accroissement du ratio $a=\tau_{t} / Y_{t}$ a les effets suivants :

(i) L'extraction de la ressource et les émissions de carbone décroissent à un rythme plus lent, de même que l'effort dans la séquestration et la séquestration elle-même (c'est-à-dire $: g_{R t}^{e}, g_{P t}^{e}$, $g_{l t}^{e}$ et $g_{Q t}^{e}$ augmentent).

(ii) L'intensité de l'effort de séquestration $\left(l_{t}^{e} / Q_{t}^{e}\right)$, l'effort par unité de contenu en carbone $\left(l_{t}^{e} / \gamma R_{t}^{e}\right)$, ainsi que le taux instantané de séquestration $\left(Q_{t}^{e} / \gamma R_{t}^{e}\right)$ augmentent.

(iii) La pollution effective par unité de contenu en carbone $\left(P_{t}^{e} / \gamma R_{t}^{e}\right)$ diminue.

(iv) L'effort dans la production de bien de consommation $\left(L_{Y}^{e}\right)$ est inchangée.

Supposons $0 \leq a \leq \nu \delta /(1-\eta) \rho$. Un accroissement du ratio $a=\tau_{t} / Y_{t}$ a deux effets principaux. Tout d'abord, la pollution devient plus coûteuse, ce qui conduit l'économie à retarder l'extraction $\left(g_{R t}^{e}\right.$ augmente). Par ailleurs, la séquestration du carbone devient plus profitable; aussi la quantité de travail par unité de contenu en carbone $\left(l_{t}^{e} / \gamma R_{t}^{e}\right)$ augmente-t-elle. Dès lors, $Q_{t}^{e} / \gamma R_{t}^{e}$, c'est-à-dire, le taux instantané de séquestration du carbone, augmente également. Simultanément, la pollution réellement émise par unité de contenu en carbone $\left(P_{t}^{e} / \gamma R_{t}^{e}\right)$ diminue. Comme la séquestration devient plus profitable, l'intensité du travail dans cette activité $\left(l_{t}^{e} / Q_{t}^{e}\right)$ augmente.

Donnons à présent quelques éléments sur les effets de court terme d'une telle politique climatique sur le niveau et le taux de croissance de l'output. Tout d'abord, puisque $g_{R t}^{e}$ augmente, les premières générations extraient moins de ressource. Etant donné que le travail consacré à 
la production n'est pas modifié, le niveau de l'output diminue pour ces générations. Deuxièmement, puisque $g_{l_{t}}^{e}$ augmente, $l_{t}^{e}$, l'effort de séquestration, décroît à court-terme. Dès lors, $L_{Y}^{e}$ étant inchangé, $L_{R D t}^{e}$ et donc $g_{A t}^{e}$ augmentent. Enfin, la croissance de l'output $\left(g_{Y t}^{e}=\right.$ $\left.\nu g_{A t}^{e}+(1-\alpha) g_{R t}^{e}-\beta\right)$ est accélérée pour les premières générations.

\section{Analyse de bien-être et politique climatique optimale}

\subsection{Conditions générales d'optimalité}

Considérons maintenant le programme du planificateur social. Ce dernier maximise (9) sous les contraintes (1)-(8). Les conditions caractéristiques de l'optimum social sont présentées dans la proposition suivante.

Proposition 4 Les conditions caractéristiques de l'optimum social sont (nous omettons les indices temporels par commodité de notation):

$$
\begin{gathered}
\rho-\frac{\dot{U}_{C}}{U_{C}}=\frac{F_{A} h_{L}}{F_{L}}+h_{A}+\frac{\dot{F}_{L}}{F_{L}}-\frac{\dot{h}_{L}}{h_{L}}, \\
\frac{F_{L}}{q_{l}}=-\frac{1}{U_{C}}\left[\int_{t}^{+\infty}\left(U_{C} F_{X}+U_{X}\right) e^{-(\rho+\theta)(s-t)} d s\right] .
\end{gathered}
$$

et

$$
U_{C} F_{R} e^{-\rho t}=\nu-\gamma\left(1-q_{\gamma R}\right) e^{-\rho t} \int_{t}^{+\infty}\left(U_{C} F_{X}+U_{X}\right) e^{-(\rho+\theta)(s-t)} d s, \quad \text { où } \nu \text { est une constante, }
$$

Preuve. Voir Annexe 2.

Dans un premier temps, notons que les conditions (27) et (20) sont identiques. En d'autres termes, la condition de Keynes Ramsey nécessaire à l'optimum social est vérifiée à l'équilibre décentralisé.

Nous avons vu, lorsque nous avons commenté la Proposition 1, que le membre gauche de la condition (28) est le coût en termes de bien d'une unité de carbone séquestrée. Le membre droit de cette équation n'est rien d'autre que le coût social, en termes de bien là aussi, d'une unité de carbone émise dans l'atmosphère. En effet, le terme $\left(U_{C} F_{X}+U_{X}\right) e^{-(\rho+\theta)(s-t)}$ est le coût social, en termes d'utilité, d'une unité de carbone émise en $t$ pour une génération à la date $s$ (d'où le terme $\left.e^{-\rho(s-t)}\right)$, tenant compte de la dégénérescence du stock de carbone (d'où le terme $\left.e^{-\theta(s-t)}\right)$. En intégrant sur l'ensemble des générations, et en divisant par l'utilité marginale de 
la consommation, on obtient bien le coût social en termes de bien d'une unité de carbone émise dans l'atmosphère.

La condition (29) est la règle d'Hotelling à l'optimum. En l'absence de pollution $(\gamma=0)$, ou/et dans le cas où les ménages et les entreprises sont indifférents au stock de carbone accumulé

$\left(U_{X}=F_{X}=0\right)$, elle s'écrirait $U_{C} F_{R} e^{-\rho t}=\nu$, ce qui implique $\rho-\dot{U}_{C} / U_{C}=\dot{F}_{R} / F_{R}$, équation qui est naturellement vérifiée à l'équilibre. Dans le cas présent, le deuxième terme du membre droit de l'équation (29) est relatif à l'externalité environnementale : c'est pour cela que, à l'équilibre décentralisé, cette condition n'est pas vérifiée en l'absence de politique climatique. Ceci vient du fait que le coût social des émissions de carbone n'est pas intégré dans le prix de la ressource. Nous revenons sur ce point dans la dernière section, où nous montrons que l'optimum peut être mis en oeuvre à l'aide d'une taxe carbone unitaire.

\subsection{Spécification et caractérisation des sentiers optimaux}

Nous reprenons ici les spécifications présentées plus haut. Nous détaillons les résultats dans l'Annexe 3, où nous donnons une caractérisation complète de la transition de l'économie. Les principaux résultats sont résumés dans la proposition 5 ci-dessous. L'indice supérieur ${ }^{o}$ caracté- $^{\prime}$ rise une variable optimale.

Proposition 5 (i) Dans le cas où les entreprises et les ménages sont sensibles à l'état de l'environnement $(\beta>0$ et $\omega>0)$, étant donnée la présence du stock de carbone $X_{t}$, l'économie est toujours en transition et converge vers le cas où la pollution n'a pas d'impact $(\beta=\omega=0)$.

(ii) Le flux d'extraction optimal, $R_{t}^{o}$, décrô̂t avec le temps (c'est-à-dire, $g_{R t}^{o}<0$ ), et ce processus est ralenti si les préférences environnementales sont strictement positives. Puisque les flux optimaux de séquestration $\left(Q_{t}^{o}\right)$ et de pollution $\left(P_{t}^{o}\right)$ sont proportionnels à $R_{t}^{o}$, ils décroissent également avec le temps.

(iii) La quantité optimale de travail dans la production, $L_{Y}^{o}$, est constante dans le temps. Le travail dans la séquestration, $l_{t}^{o}$, est proportionnel au flux d'extraction, $R_{t}^{o}$, et suit donc la même dynamique (c'est-à-dire $g_{l t}^{o}=g_{R t}^{o}$ ). Ainsi, le travail dans la recherche, $L_{R D t}^{o}$, crồt dans le temps et converge vers $1-L_{Y}^{o}$ à l'infini.

Les niveaux et taux de croissance optimaux des différentes variables sont donnés dans l'Annexe 3 .

Preuve. Voir Annexe 3.

Analysons la trajectoire de l'économie à l'optimum social. Si l'on considère le cas où le stock de carbone n'affecte ni la production des entreprises ni l'utilité des ménages, c'est-à-dire le cas 
où $\beta=\omega=0$, l'économie saute immédiatement à l'état-stationnaire que nous avons décrit après la Proposition 2 (lorsque $\tau=0$ à l'équilibre décentralisé). En résumé, $l^{o}=0, Q^{o}=0$, et $L_{R D}^{o}$ ainsi que $L_{Y}^{o}$ sont des constantes : aucune activité de séquestration n'est menée, et les efforts de production et de $R \& D$ sont constants. Par ailleurs, $g_{R}^{o}=-\rho$ et $P_{t}^{o}=\gamma R_{t}^{o}$ : le contenu en carbone de chaque unité de ressource est émis en totalité. Enfin, on obtient à partir de la formule (40) de l'Annexe 3, que le taux de croissance de l'output, $g_{Y}^{o}$, est égal à $\nu \delta-\rho$, comme dans les modèles de croissance endogène avec ressources non-renouvelables non polluantes (voir par exemple Grimaud et Rouge, 2003).

Considérons maintenant le cas plus général où $\beta>0$ et $\omega>0$. Contrairement au cas précédent, l'économie est ici toujours en transition. L'expression de $R_{t}^{o}$ donnée dans l'annexe 3 montre bien que l'extraction de la ressource diminue au fil du temps, mais $g_{R t}^{o}$ est maintenant supérieur à $-\rho$. En d'autres termes, quand la pollution atmosphérique affecte l'utilité des ménages ainsi que la productivité des entreprises, le planificateur social retarde l'extraction de la ressource (voir Withagen, 1994, pour un résultat similaire dans un contexte d'équilibre partiel). Les trajectoires d'extraction de la ressource à l'optimum social et à l'équilibre décentralisé sont représentées dans la Figure 1. Puisque les variables $l_{t}^{o}$, $Q_{t}^{o}$ et $P_{t}^{o}$ sont des fonctions linéaires de $R_{t}^{o}$, elles ont la même dynamique : elles décroissent au fil du temps, de même que leurs taux de croissance. Cela implique évidemment que la part séquestrée des émissions $\left(Q_{t}^{o} / P_{t}^{o}\right)$ reste constante dans le temps.

Observons que $L_{Y}^{o}$ est également constant (voir annexe 3a). Dès lors, le flux de travail restant est réparti entre séquestration et recherche. Puisque $l_{t}^{o}$ décroît au fil du temps, $L_{R D t}^{o}$ croît : alors que l'effort de séquestration devient de plus en plus faible, l'investissement dans la $R \& D$ augmente.

Lorsque $t$ tend vers l'infini, les taux $g_{R t}^{o}=g_{l_{t}}^{o}=g_{Q_{t}}^{o}=g_{P_{t}}^{o}$ tendent vers $-\rho$. Simultanément, $l_{t}^{o}$ diminue progressivement et tend vers $0, L_{R D t}^{o}$ tend vers $1-\alpha \rho / \delta \nu$, et $g_{Y t}^{o}$ tend vers $\nu \delta-\rho-\beta$. Ces valeurs asymptotiques sont identiques, à l'exception de $g_{Y t}^{o}$, à celles de l'état-stationnaire présenté plus haut, lorsque $\beta=\omega=0$. La ressource est asymptotiquement épuisée, et les émissions de carbone deviennent nulles à l'infini. C'est la raison pour laquelle, la trajectoire socialement optimale de l'économie converge asymptotiquement vers l'état-stationnaire de l'économie dans laquelle il n'y a pas de pollution (pour une illustration des résultats concernant les trajectoires de l'extraction de la ressource, du stock de carbone, et de l'output, voir les Figures 1, 2 et 3). 


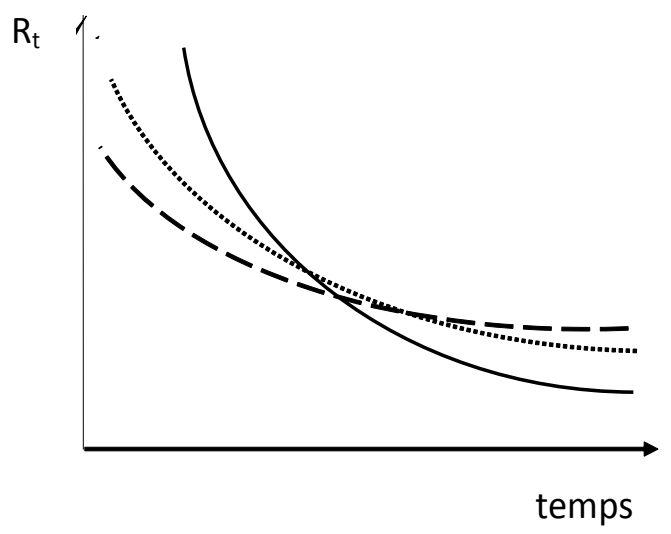

Figure 1 : Extraction de la ressource

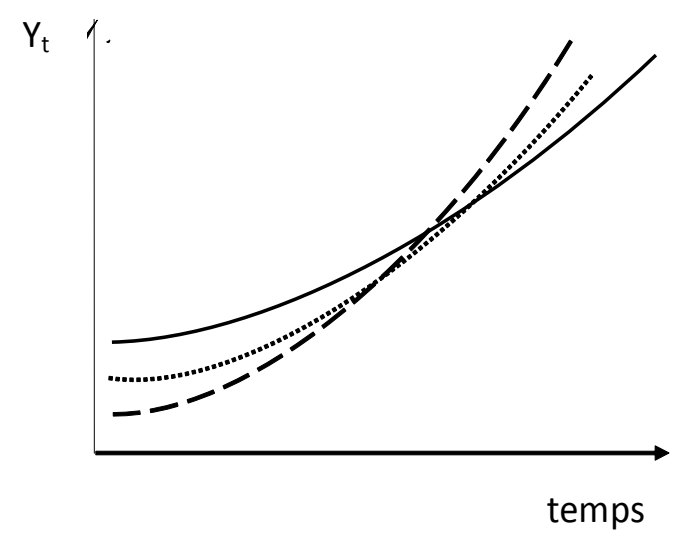

Figure 3 : Production

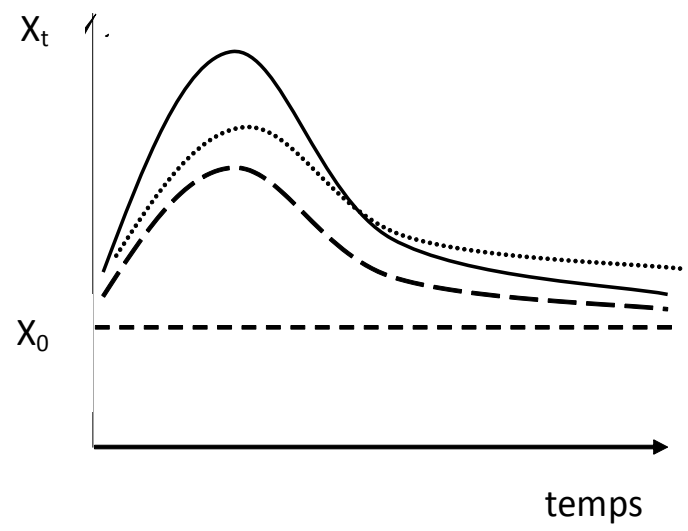

Figure 2 : Stock de carbone

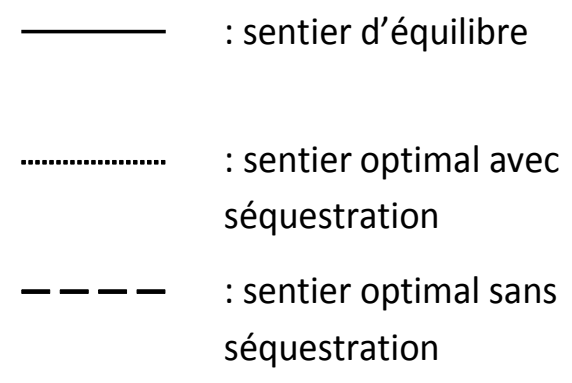




\subsection{Impact de la séquestration sur les sentiers optimaux}

Appelons $Z_{t}^{o \varnothing}$ le niveau optimal de toute variable $Z_{t}$ dans le cas où une technologie de séquestration n'est pas disponible. Nous donnons les niveaux et les taux de croissance optimaux des différentes variables dans les Annexes 3 et 4.

Comparer l'optimum social dans le cas présent avec l'optimum social présenté plus haut (avec séquestration), conduit à la proposition suivante.

Proposition 6 Introduire une technologie de séquestration du carbone modifie les sentiers optimaux de la façon suivante :

(i) L'extraction de la ressource est plus rapide (c'est-à-dire, $\left.g_{R t}^{o}<g_{R t}^{o \varnothing}\right)$ : on extrait plus de ressource lors des phases initiales, et moins dans le futur.

(ii) Les effets de court terme sur la pollution peuvent être différents de ceux de long terme. A court terme, l'augmentation de l'extraction de la ressource (voir (i) plus haut) stimule les émissions de $\mathrm{CO}_{2}$, tandis que la séquestration a l'effet inverse : l'effet total est ambigu. A long terme, puisque le flux d'extraction diminue (voir (i) plus haut) et qu'une part du carbone est séquestrée, les émissions de carbone diminuent sans ambigüité.

(iii) La croissance de l'output est plus faible (c'est-à-dire, $\left.g_{Y t}^{o}<g_{Y t}^{o \varnothing}\right)$.

Preuve. Pour la caractérisation de l'optimum social en l'absence d'une technologie de séquestration, voir l'Annexe 4.

Comparons le taux de croissance de l'extraction de la ressource à l'équilibre décentralisé $\left(g_{R}^{e}\right)$ en l'absence de politique climatique à son niveau optimal. En combinant les résultats obtenus dans les propositions 2 et 6 , nous obtenons les inégalités suivantes :

$$
g_{R}^{e}=-\rho<g_{R t}^{o}<g_{R t}^{o \varnothing},
$$

qui sont illustrées dans la Figure 1. Tout d'abord, $g_{R}^{e}<g_{R t}^{o \varnothing}$ signifie que, dans une économie dans laquelle il n'y a pas de possibilité de séquestrer le carbone, l'extraction de la ressource dans un régime de laissez-faire est trop rapide par rapport au rythme optimal. On peut trouver un résultat similaire dans un contexte d'équilibre partiel dans Withagen, 1994. La prise en compte dans l'analyse de la possibilité de séquestrer une partie des émissions de carbone permet d'obtenir deux résultats complémentaires. L'inégalité $g_{R}^{e}=-\rho<g_{R t}^{o}$ est une extension du résultat précédent : même s'il est possible de stocker du carbone, il est optimal de retarder l'extraction, par rapport à ce qui a lieu dans le laissez-faire. Cependant, l'inégalité $g_{R t}^{o}<g_{R t}^{o \varnothing}$ établit que, en présence d'une technologie de séquestration, le sentier optimal d'extraction est 
moins contraignant qu'en l'absence de cette technologie. En d'autres termes, si, dans les modèles standards avec ressources non-renouvelables, l'extraction optimale est moins rapide quand la pollution est prise en compte, ici, la séquestration du carbone permet de relâcher partiellement la contrainte environnementale. Comme nous le mentionnons plus haut, le sacrifice des premières générations est réduit.

Comme nous le précisons dans la proposition ci-dessus, l'impact de la séquestration du carbone sur le sentier de pollution optimal est moins clair. Considérons tout d'abord les premières générations. Deux effets de sens contraires influent sur le sentier de pollution : un effet-extraction, et un effet-séquestration. Puisque l'extraction de la ressource augmente, les émissions de carbone ont également tendance à augmenter; néanmoins, dans le même temps, l'activité de séquestration réduit la pollution. La question est donc : quel est l'effet dominant? Ceci dépend des paramètres du modèle qui apparaissent dans le terme entre parenthèses dans l'expression de $P^{o}$ (voir annexe 3). En particulier, on peut vérifier que, pour des valeurs élevées de $\beta+\omega$, l'effet-séquestration tend à l'emporter. Ceci signifie que, lorsque les entreprises et les ménages sont très sensibles à la qualité de l'environnement, la séquestration est intensive, et donc la pollution diminue, malgré l'augmentation du flux d'extraction. Dans ce cas, la séquestration réduit le niveau de pollution optimal pour les premières générations. En revanche, si $\beta+\omega$ est faible, c'est-à-dire, si les entreprises et les ménages sont peu sensibles au stock de carbone accumulé dans l'atmosphère, l'effet-extraction domine l'effet-séquestration : la pollution augmente car l'activité de séquestration est faible. Nous nous trouvons donc dans le cas contre-intuitif où la possibilité de séquestrer le carbone conduit à une augmentation simultanée de l'extraction de la ressource et des émissions de carbone pour les premières générations.

A long-terme, la séquestration du carbone conduit sans ambigüité à des émissions plus faibles pour les générations futures. En effet, nous avons montré que le flux d'extraction diminue; dès lors, indépendamment de la sensibilité des agents au stock de carbone, et indépendamment des quantités séquestrées, les émissions diminuent.

Etudions maintenant l'effet de la séquestration sur la croissance optimale. Tout d'abord, $l^{\circ \varnothing}$ et $Q^{o \varnothing}$ sont évidemment nuls. Ceci implique $L_{R D t}^{o}<L_{R D t}^{o \varnothing}$ : la quantité de travail allouée à la $R \& D$ est plus importante dans le cas sans séquestration, puisqu'il n'y a pas de travail dans la séquestration. Il y a donc un premier effet-recherche, qui est défavorable à la croissance. En outre, l'effet-extraction analysé ci-dessus a également un effet négatif sur la croissance. En d'autres termes, les deux inégalités $L_{R D t}^{o}<L_{R D t}^{o \varnothing}$ (et donc $g_{A t}^{o}<g_{A t}^{o \varnothing}$ ) et $g_{R t}^{o}<g_{R t}^{o \varnothing}$ mènent immédiatement à la suivante $: g_{Y t}^{o}=\nu \delta L_{R D t}^{o}+(1-\alpha) g_{R t}^{o}-\beta<g_{Y t}^{o \varnothing}=\nu \delta L_{R D t}^{o \varnothing}+(1-\alpha) g_{R t}^{o \varnothing}-\beta$. 
La séquestration du carbone a donc un effet négatif sur la croissance économique.

En résumé, la technologie de séquestration du carbone permet d'alléger la contrainte environnementale : en présence d'une technologie de séquestration du carbone, les premières générations extraient davantage de ressource et consomment plus à l'optimum. En d'autres termes, leur "sacrifice" est réduit (voir les Figures 1 et 3).

\subsection{Politique climatique optimale}

En comparant les conditions présentées dans les propositions 1 et 4, nous obtenons l'expression générale de la taxe carbone optimale. En utilisant ensuite les spécifications (23)-(26), nous en donnons une expression simple.

Proposition 7 La taxe carbone optimale est:

$$
\tau_{t}^{o}=-\frac{1}{U_{C}}\left[\int_{t}^{+\infty}\left(U_{C} F_{X}+U_{X}\right) e^{-(\rho+\theta)(s-t)} d s\right]
$$

Dans le cas spécifié, elle prend la forme suivante:

$$
\tau_{t}^{o}=\frac{\beta+\omega}{\rho+\theta} Y_{t}
$$

Comme nous l'avons mentionné précédemment, le niveau de la taxe importe ici, contrairement aux résultats standards de la littérature (voir Sinclair, 1994, Grimaud et Rouge, 2005, 2008, Groth et Schou, 2007, par exemple). Faisons quelques commentaires sur l'expression générale de cette taxe optimale. Tout d'abord, le terme $U_{C} F_{X}+U_{X}$ indique bien que les ménages sont affectés par l'émission d'une unité de carbone de deux façons : directement par la baisse de leur utilité (d'où le terme $U_{X}$ ), et indirectement par la baisse de la production (d'où le terme $U_{C} F_{X}$ ). D'autre part, comme nous l'avons montré dans les commentaires de la proposition 4, la taxe optimale est le coût social en termes de bien d'une unité de carbone émise dans l'atmosphère.

Dans sa forme spécifiée, la taxe optimale exprimée en termes d'utilité, $((\beta+\omega) /(\rho+\theta))$, est constante. En revanche, c'est une fonction croissante du temps quand elle est exprimée en termes de bien. En effet, la croissance économique étant positive, l'utilité marginale de la consommation décroît dans le temps : $U_{C}$ diminue, et donc $1 / U_{C}$ augmente. Par ailleurs, notons que cette taxe carbone est une fonction croissante des paramètres $\beta$ et $\omega$, qui représentent l'impact négatif de la pollution sur la productivité des entreprises et l'utilité des ménages. Elle est également une fonction décroissante du taux de préférence pour le présent, $\rho$ : plus on donne de poids aux 
générations présentes, plus le niveau de la taxe est faible. De même, elle décroît avec le taux de dégénérescence du stock de carbone, $\theta$.

Lorsque la technologie de séquestration du carbone est disponible, le planificateur social doit donner aux entreprises le bon signal en termes de coût social de la pollution, afin de les inciter à un effort de séquestration optimal. Dans le cas non spécifié, on a $\tau_{t}^{o}=F_{L} / q_{l}$ : la taxe carbone optimale est égale à la quantité de bien à laquelle renoncent les entreprises pour séquestrer une unité de carbone supplémentaire. Formellement, dans ce modèle, ceci se fait à travers un transfert de travail entre les secteurs du bien de consommation et de la séquestration.

Nous avons expliqué précédemment pourquoi il est nécessaire de taxer directement le carbone (et non la ressource) pour atteindre l'optimum. Nous pouvons néanmoins relier nos résultats à la littérature standard, c'est-à-dire sans séquestration. Nous montrons ci-dessous que la taxe carbone optimale présentée dans la proposition 7 peut être réinterprétée ex-post comme une taxe ad-valorem décroissante sur la ressource naturelle.

Le prix "total" payé par l'entreprise pour la ressource est : $p_{R} R+\tau^{o}\left[\gamma R-(\gamma R)^{\eta} l^{1-\eta}\right]$. En utilisant l'expression de $l^{e}$ donnée dans (30) et $\tau^{o}=(\beta+\omega) Y /(\rho+\theta)$ (voir proposition 7), ce prix est donné par :

$$
p_{R} R\left[1+\frac{\gamma(\beta+\omega) Y}{(\rho+\theta) p_{R}}\left(1-\left(\frac{(\beta+\omega) \rho(1-\eta)}{\nu \delta(\rho+\theta)}\right)^{(1-\eta) / \eta}\right)\right]
$$

Puisque $g_{Y}=r-\rho$, et $g_{p_{R}}=r$, le rapport $Y / p_{R}$ décroît au cours du temps. Donc, la formule précédente peut s'écrire $p_{R} R(1+\sigma)$, où $\sigma$ peut être interprété comme une taxe ad valorem décroissante sur la ressource.

\section{Conclusion}

Nous avons présenté un modèle de croissance endogène (à la Romer) dans lequel un bien de consommation est produit à partir d'un stock de connaissance, de travail et d'une ressource fossile (non-renouvelable et polluante). L'utilisation de cette ressource génère des émissions de $\mathrm{CO}_{2}$ dont le stock accumulé dans l'atmosphère affecte négativement à la fois l'utilité des ménages et la productivité des entreprises. Le principal objectif de cet article était d'étudier en quoi les résultats précédents de la littérature sont modifiés et étendus lorsque la possibilité de séquestrer tout ou partie du carbone émis lors de la combustion de la ressource est prise en compte dans l'analyse. Ceci implique que, contrairement à ce qui est implicitement admis dans la littérature 
standard, la pollution est partiellement dissociée de l'extraction de la ressource.

Nous avons caractérisé les trajectoires de l'équilibre dans l'économie décentralisée. Ces trajectoires dépendent notamment de la politique climatique que nous mettons en place. Celle-ci consiste ici en une taxe unitaire sur les émissions de carbone. En effet, dans notre contexte, il n'est pas équivalent de taxer les émissions de carbone et la ressource. Nous avons alors étudié l'impact de cette taxe sur les trajectoires des variables de l'économie.

Nous avons également caractérisé les trajectoires optimales. Nous montrons que la technologie de séquestration du carbone accélère l'utilisation optimale de la ressource, et permet donc de relâcher partiellement la contrainte environnementale, ce qui réduit le sacrifice des premières générations. De plus, elle modifie le sentier d'émissions de $\mathrm{CO}_{2}$ : si celles-ci diminuent sans ambigüité à long terme, il se peut qu'elles augmentent pour les premières générations, si la sensibilité des ménages et des entreprises au stock de carbone est faible. Enfin, nous montrons que l'utilisation de cette technologie a un impact négatif sur la croissance économique.

Nous avons enfin déterminé la politique climatique optimale. Tout d'abord, nous montrons que le niveau de la taxe importe ici : il correspond en fait au coût social en termes de bien d'une unité de carbone émise dans l'atmosphère. Dès lors, c'est bien lui qui génère les incitations à un effort optimal de séquestration, puisqu'il est aussi égal à la quantité de bien à laquelle renoncent les entreprises pour séquestrer une unité de carbone supplémentaire. Par ailleurs, la taxe carbone optimale croît dans le temps et conduit à retarder l'extraction de la ressource. Enfin, on peut l'interpréter, ex-post, comme une taxe ad-valorem décroissante sur la ressource : cette politique climatique diminue le taux de croissance du prix "total" de la ressource (c'est-à-dire le prix de la ressource, taxe-carbone comprise).

On peut envisager plusieurs extensions possibles à ce modèle. En premier lieu, nous pourrions prendre en compte l'effet du progrès technique dans l'activité de séquestration. Par ailleurs, il serait utile de considérer l'existence d'une source d'énergie renouvelable et non polluante (voir sur ce thème, dans un modèle sans séquestration, Grimaud et Rouge, 2008, par exemple). Cela permettrait notamment d'étudier l'impact de la séquestration du carbone sur le rythme auquel les énergies propres se substituent aux ressources fossiles. Nous pouvons penser que la possibilité de séquestrer le carbone va ralentir le recours aux énergies propres. En ce qui concerne la production d'électricité, elle pourrait même relancer l'utilisation de centrales à charbon, au détriment du gaz naturel, donnant ainsi lieu à une renaissance du charbon (Newell et al., 2006) sur les prochaines décennies. 


\section{Annexe}

\section{Annexe 1 : Caractérisation de l'équilibre décentralisé dans le cas spécifié}

Appliquons les spécifications (23)-(26) présentées dans la section 3.3 aux trois conditions caractéristiques de l'équilibre décentralisé (20), (21) et (22) de la proposition 1.

a) Condition (20) :

Puisque $U_{C}=1 / C$, on a $\dot{U}_{C} / U_{C}=-g_{C}=-g_{Y}$. D'autre part, $F_{A} h_{L} / F_{L}=\nu \delta L_{Y} / \alpha$. On a aussi $h_{A}=\delta L_{R D}=g_{A}$. Enfin, puisque $F_{L}=\alpha Y / L_{Y}$, on a $\dot{F}_{L} / F_{L}=g_{Y}-g_{L_{Y}}$ et, puisque $h_{L}=\delta A$, on obtient $\dot{h}_{L} / h_{L}=g_{A}$.

En substituant ces résultats dans l'équation de Keynes-Ramsey (20), on obtient l'équation différentielle de Ricatti $\dot{L}_{Y}=-\rho L_{Y}+(\nu \delta / \alpha) L_{Y}^{2}$, dont la solution est $\frac{1}{e^{\rho t}\left[\frac{1}{L_{Y}}-\frac{\nu \delta}{\alpha \rho}\right]+\frac{\nu \delta}{\alpha \rho}}$. En utilisant la condition de transversalité du calcul du consommateur, on peut montrer que la variable $L_{Y}$ saute immédiatement sur sa valeur d'état stationnaire : $L_{Y}^{e}=\frac{\alpha \rho}{\nu \delta}$.

b) Condition (21) :

On a $F_{L}=\alpha Y / L_{Y}=\nu \delta Y / \rho$, en utilisant le résultat précédent. D'autre part, $q_{l}=(1-$ $\eta) Q / l=(1-\eta)(\gamma R / l)^{\eta}$. En substituant ces résultats dans l'équation (21), on obtient $l=$ $\left[\frac{(1-\eta) \rho \tau}{\nu \delta Y}\right]^{1 / \eta} \gamma R, Q=\left[\frac{(1-\eta) \rho \tau}{\nu \delta Y}\right]^{(1-\eta) / \eta} \gamma R$, et $P=\left[1-\left(\frac{(1-\eta) \rho \tau}{\nu \delta Y}\right)^{(1-\eta) / \eta}\right] \gamma R$.

c) Condition (22) :

Nous avons $F_{R}=(1-\alpha) Y / R$, et $q_{\gamma R}=\eta Q / \gamma R$. La condition (22) devient $(1-\alpha) Y / R-$ $p_{R}=\tau \gamma\left[1-\eta\left(\frac{(1-\eta) \rho \tau}{\nu \delta Y}\right)^{(1-\eta) / \eta}\right]$. Puisque $\dot{p}_{R} / p_{R}=r$, on a $p_{R}=p_{R_{0}} e^{\int_{0}^{t} r_{u} d u}$. D'autre part, la condition $\rho-\dot{U}_{C} / U_{C}=r$ devient $\dot{C} / C=r-\rho$, ce qui donne $C=C_{0} e^{\int_{0}^{t}\left(r_{u}-\rho\right) d u}$. En utilisant ces résultats, la condition (22) s'écrit : $R=\frac{1-\alpha}{p_{R_{0}} e^{\rho t} / C_{0}+\frac{\tau \gamma}{Y}\left[1-\eta\left(\frac{(1-\eta) \rho \tau}{\nu \delta Y}\right)^{(1-\eta) / \eta}\right]}$, où la constante $p_{R_{0}} / C_{0}$ est solution de $\int_{0}^{\infty} R_{t} d t=S_{0}$.

Résumé des résultats :

$$
\begin{gathered}
L_{Y}^{e}=\frac{\alpha \rho}{\nu \delta}, \quad l_{t}^{e}=\left[\frac{(1-\eta) \rho \tau}{\nu \delta Y}\right]^{1 / \eta} \gamma R_{t}^{e}, \quad L_{R D t}^{e}=1-L_{Y t}^{e}-l_{t}^{e}, \\
R_{t}^{e}=\frac{1-\alpha}{\left(p_{R_{0}} / C_{0}\right) e^{\rho t}+\frac{\tau \gamma}{Y}\left[1-\eta\left(\frac{(1-\eta) \rho \tau}{\nu \delta Y}\right)^{(1-\eta) / \eta}\right]}, \text { avec } \int_{0}^{\infty} R_{t} d t=S_{0} . \\
Q_{t}^{e}=\left[\frac{(1-\eta) \rho \tau}{\nu \delta Y}\right]^{(1-\eta) / \eta} \gamma R_{t}^{e}, \quad P_{t}^{e}=\left[1-\left(\frac{(1-\eta) \rho \tau}{\nu \delta Y}\right)^{(1-\eta) / \eta}\right] \gamma R_{t}^{e},
\end{gathered}
$$




$$
g_{A t}^{e}=\delta L_{R D t}^{e}, \quad g_{R t}^{e}=g_{l_{t}}^{e}=g_{Q t}^{e}=g_{P t}^{e}=\frac{-\rho}{1+\frac{\tau \gamma C_{0}}{p_{R 0} Y}\left[1-\eta\left(\frac{(1-\eta) \rho \tau}{\nu \delta Y}\right)^{(1-\eta) / \eta}\right] e^{-\rho t}}, \quad g_{Y t}^{e}=\nu g_{A t}^{e}+(1-\alpha) g_{R t}^{e}-\beta
$$

\section{Annexe 2 : Analyse de bien-être dans le cas non spécifié}

Le Hamiltonien du programme du planificateur social est :

$H=U\left[F\left(A, 1-L_{R D}-l, R, X\right), X\right] e^{-\rho t}+\mu h\left(A, L_{R D}\right)-\nu R+\zeta\left[\theta\left(X_{0}-X\right)+(\gamma R-q(\gamma R, l))\right]$,

où $\mu, \nu$ et $\zeta$ sont les variables adjointes associées aux trois contraintes. Les conditions du premier ordre $\partial H / \partial L_{R D}=0, \partial H / \partial l=0$ et $\partial H / \partial R=0$ s'écrivent respectivement :

$$
\begin{gathered}
-U_{C} F_{L} e^{-\rho t}+\mu h_{L}=0, \\
-U_{C} F_{L} e^{-\rho t}-\zeta q_{l}=0, \\
U_{C} F_{R} e^{-\rho t}-\nu+\zeta \gamma\left(1-q_{\gamma R}\right)=0 .
\end{gathered}
$$

De plus, $\partial H / \partial A=-\dot{\mu}, \partial H / \partial S=-\dot{\nu}$ et $\partial H / \partial X=-\dot{\zeta}$ s'écrivent respectivement

$$
\begin{gathered}
U_{C} F_{A} e^{-\rho t}+\mu h_{A}=-\dot{\mu}, \\
-\dot{\nu}=0, \\
\left(U_{C} F_{X}+U_{X}\right) e^{-\rho t}-\zeta \theta=-\dot{\zeta} .
\end{gathered}
$$

a) Condition de Keynes-Ramsey.

En log-différenciant l'équation (34), on obtient $\dot{U}_{C} / U_{C}+\dot{F}_{L} / F_{L}-\rho=g_{\mu}+\dot{h}_{L} / h_{L}$. Par ailleurs, de l'équation (37), on tire $-g_{\mu}=U_{C} F_{A} e^{-\rho t} / \mu+h_{A}$, où $\mu$ est obtenu à partir de (34). En éliminant $g_{\mu}$, on obtient la condition de Keynes-Ramsey (27).

b) Détermination de la variable adjointe $\zeta_{t}$.

En intégrant l'équation différentielle (39), on obtient $\zeta_{t}=e^{\theta t}\left[\zeta_{0}-\int_{0}^{t}\left(U_{C} F_{X}+U_{X}\right) e^{-(\rho+\theta) s} d s\right]$. La condition de transversalité $\lim _{t \rightarrow \infty} \zeta_{t} X_{t}=0$ conduit à $\zeta_{0}=\int_{0}^{\infty}\left(U_{C} F_{X}+U_{X}\right) e^{-(\rho+\theta) s} d s$. En substituant ce résultat dans l'équation précédente, on obtient $\zeta_{t}=e^{-\rho t} \int_{t}^{\infty}\left(U_{C} F_{X}+U_{X}\right) e^{-(\rho+\theta)(s-t)} d s$.

c) Condition de Hotelling.

En remplaçant $\zeta_{t}$ dans (36) par son expression obtenue dans b) ci-dessus, on obtient la condition de Hotelling (29).

d) Séquestration optimale. 
De la condition (35), on tire $\zeta_{t}=-U_{C} F_{L} e^{-\rho t} / q_{l}$, ce qui conduit immédiatement à la condition (28).

\section{Annexe 3 : Analyse de bien-être dans le cas spécifié}

a) Condition de Keynes-Ramsey.

Cette équation est la même à l'équilibre décentralisé et à l'optimum social. Elle conduit donc à la même équation différentielle de Ricatti (voir Annexe 1), dont la solution est $L_{Y}^{o}=\alpha \rho / \delta \nu$.

b) Travail dans l'activité de séquestration, carbone séquestré et pollution émise.

En utilisant les spécifications (23)-(26), la condition (28) s'écrit : $[\nu \delta / \rho(1-\eta)](l / \gamma R)^{1 / \eta} \eta Y=$ $[(\beta+\omega) /(\rho+\theta)] Y$, ce qui donne

$$
l^{o}=\left[\frac{\rho(1-\eta)(\beta+\omega)}{\nu \delta(\rho+\theta)}\right]^{1 / \eta} \gamma R^{o} .
$$

On en déduit

$$
Q^{o}=\left(\gamma R^{o}\right)^{\eta} l^{o(1-\eta)}=\left[\frac{\rho(1-\eta)(\beta+\omega)}{\nu \delta(\rho+\theta)}\right]^{(1-\eta) / \eta} \gamma R^{o}
$$

et

$$
P^{o}=\gamma R^{o}-Q^{o}=\left[1-\left[\frac{\rho(1-\eta)(\beta+\omega)}{\nu \delta(\rho+\theta)}\right]^{(1-\eta) / \eta}\right] \gamma R^{o} .
$$

c) Flux d'extraction de la ressource.

Avec les spécifications (23)-(26), l'équation (29) s'écrit : $(1-\alpha) / R=\nu e^{\rho t}+\gamma\left[1-\left[\frac{\rho(1-\eta)(\beta+\omega)}{\nu \delta(\rho+\theta)}\right]^{(1-\eta) / \eta}\right] \frac{\beta+\omega}{\rho+\theta}$. On en déduit le flux d'extraction :

$$
R^{o}=\frac{1-\alpha}{\nu e^{\rho t}+\frac{\gamma(\beta+\omega)}{\rho+\theta}\left[1-\left[\frac{\rho(1-\eta)(\beta+\omega)}{\nu \delta(\rho+\theta)}\right]^{(1-\eta) / \eta}\right]}
$$

où $\nu$ est solution de $\int_{0}^{\infty} R_{t}^{o} d t=S_{0}$.

Les taux de croissance sont :

$$
g_{A}^{o}=\delta L_{R D}^{o}, \quad g_{R}^{o}=g_{l}^{o}=g_{Q}^{o}=g_{P}^{o}=\frac{-\rho}{1+\frac{\gamma(\beta+\omega)}{\nu(\rho+\theta)}\left[1-\left[\frac{\rho(1-\eta)(\beta+\omega)}{\nu \delta(\rho+\theta)}\right]^{(1-\eta) / \eta}\right] e^{-\rho t}}, \quad g_{Y}^{o}=\nu g_{A}^{o}+(1-\alpha) g_{R}^{o}-\beta
$$




\section{Annexe 4 : Analyse de bien-être sans séquestration}

S'il n'existe pas de technologie de séquestration du carbone, la maximisation du bien-être conduit aux résultats suivants (nous rappelons ici que nous notons $X_{t}^{o \varnothing}$ le niveau optimal de toute variable $X_{t}$ dans ce cas de figure) :

$$
\begin{gathered}
L_{Y}^{o \varnothing}=\alpha \rho / \delta \nu, L_{A}^{o \varnothing}=1-(\alpha \rho / \delta \nu), R_{t}^{o \varnothing}=\frac{1-\alpha}{\varphi_{0}^{\varnothing} e^{\rho t}+B^{\varnothing}}, g_{R}^{o \varnothing}=\frac{-\rho}{1+B^{\varnothing} / \varphi_{0}^{\varnothing} e^{\rho t}}, g_{A}^{o \varnothing}=\delta L_{A}^{o \varnothing}, \\
g_{Y}^{o \varnothing}=\nu \delta L_{A}^{o \varnothing}+(1-\alpha) g_{R}^{o \varnothing}-\beta, \text { où } \varphi_{0}^{\varnothing}=\frac{B^{\varnothing}}{e^{\left(B^{\varnothing} \rho S_{0} /(1-\alpha)\right)}-1} \text { et } B^{\varnothing}=\gamma(\beta+\omega) /(\rho+\theta) .
\end{gathered}
$$




\section{Bibliographie}

Aghion, P., et Howitt, P., Endogenous Growth Theory, The MIT Press, 1998.

Barro, R. et Sala-i-Martin X. (2003). "Economic Growth", The MIT Press.

J.P. Bénassy (1998), "Is there always too little research in endogenous growth with expanding product variety?", European Economic Review, vol. 42, pp 61-69.

Gerlagh, R., ITC in a global growth-climate model with CCS : The value of induced technical change for climate stabilization, The Energy Journal, Special issue on endogenous technological change and the economics of atmospheric stabilization (2006) 223-240.

Gerlagh, R., van der Zwaan, B.C.C., Options and instruments for a deep cut in CO2 emissions : Carbon capture or renewables, taxes or subsidies? The Energy Journal 27 (2006) 25-48.

Grimaud, A., Rouge, L., Non-renewable resources and growth with vertical innovations : optimum, equilibrium and economic policies, J. Environ. Econom. Management 45 (2003) 433453.

Grimaud, A., Rouge, L., Polluting Non-Renewable Resources, Innovation and Growth : Welfare and Environmental Policy, Resource and Energy Economics 27(2) (2005) 109-129.

Grimaud A., Rouge, L., Environment, Directed Technical Change and Economic Policy, Environmental and Resource Economics, Forthcoming.

Groth, C., Schou, P., Growth and Non-Renewable Resources : The Different Roles of Capital and Resource Taxes, J. Environ. Econom. Management 53(1) (2007) 80-98.

Hart, R., Growth, Environment annd Innovation - A Model with Production Vintages and Environmentally Oriented Research, J. Environ. Econom. Management 48 (2004)1078-1098.

Hoffert, M., et al., Advanced Technology Paths to Global Climate Stability : Energy for a Greenhouse Planet, Science 298 (2002) 981-87.

International Energy Agency (IEA), Energy Technology Perspectives, IEA Publications, Paris, 2006.

IPCC, Special Report on Carbon dioxide Capture and Storage, Contribution of Working Group III, Report of the Intergovernmental Panel on Climate Change, Cambridge University Press, Cambridge, UK, 2005.

Jones, C.I., Williams, J.C., Measuring the Social Returns to R\&D, Quarterly Journal of Economics 113 (1998) 1119-1135.

Kolstad, C.D., Toman, M., The Economics of Climate Policy, Resources For the Future, Discussion Paper \\#00-40REV (2001).

Newell, R.G., Jaffe, A.B., Stavins, R.N., The effects of economic and policy incentives on 
carbon mitigation technologies, Energy Economics, 28(5-6) (2006) 563-578.

Popp, D., ENTICE : Endogenous technological change in the DICE model of global warming, J. Environ. Econom. Management 48 (2004) 742-768.

Schou, P., Polluting non-renewable resources and growth, Environmental and Resource Economics 16 (2000) 211-227.

Schou, P., When environmental policy is superfluous : growth and polluting resources, Scandinavian Journal of Economics 104 (2002) 605-620.

Sinclair, P., High does nothing and rising is worse : carbon taxes should keep declining to cut harmful emissions, The Manchester School 60(1) (1992) 41-52.

Sinclair, P., On the optimum trend of fossil fuel taxation, Oxford Economic Papers 46 (1994) 869-877.

Smulders, S., Gradus, R., Pollution abatement and long-term growth, European Journal of Political Economy, 12(3), (1996) 505-32.

Tahvonen, O., Fossil Fuels, Stock Externalities, and Backstop Technology, Canadian Journal of Economics XXX 4a, (1997) 855-874.

Ulph, A., Ulph, D., The optimal time path of a carbon tax, Oxford Economic Papers 46 (1994) 857-868.

Withagen, C., Pollution and Exhaustibility of fossil fuels, Resource and Energy Economics 16 (1994) 235-242. 